\title{
Erfrischend einfach
}

\author{
Mit Wasserflaschen die obere Extremität trainieren Reichen, greifen, manipulieren - \\ drei elementare Aktivitäten, die Patienten mit neurologischen Erkrankungen häufig trainie- \\ ren müssen. Durch alltagsnahes und intensives Üben können sie Schlüsselkomponenten wie \\ die Handgelenkextension und die Flexion im Schultergelenk verbessern. Eine gute und \\ einfache Therapie ist das Training mit Wasserflaschen.
}

$\rightarrow$ Virtuelle Realität und robotergestützte Therapie - in der Neuroreha steigt die Zahl technologischer Innovationen. Und das ist gut so, rechnet man doch in den nächsten Jahren mit einem erhöhten Patientenaufkommen und steigendem Rehabedarf. Doch auch ohne Hightech können Menschen nach Schlaganfall effektiv trainieren - handelsübliche Wasserflaschen reichen dafür als Trainingsgerät oft aus.

Das Flaschentraining orientiert sich in seinen zentralen Inhalten an den Prinzipien, die die Physiotherapeutinnen und Wissenschaftlerinnen Janet H. Carr und Roberta B. Shepherd [1, 2] gemeinsam mit Ergotherapeutin Annie McCluskey [3] für die Analyse und Therapie der oberen Extremtität (OE) nach Schlaganfall entwickelt haben. Die Therapeutinnen sprechen von einem sogenannten Bezugsrahmen, der als Hauptaktivitäten der OE das Reichen, Greifen und Manipulieren definiert. Um diese Bewegungen erfolgreich durchzuführen, legten sie für jede der Bewegungen Schlüsselkomponenten fest ( $\odot$ HINTERGRUNDWISSEN). Diese festzulegen, kann dem Therapeuten helfen, schnell und gezielt Arm- und Handbewegungen des Patienten zu analysieren und anschließend spezifische Interventionen zu entwickeln. Damit der Patient seine Fähigkeit im Reichen verbessert, soll er beispielsweise die aktive und passive Außenrotation und die Flexion im Schultergelenk trainieren. Carr, Shepherd und McCluskey heben hervor, dass man die Therapie auf die Muskelschwäche und die Koordination (Minussymptome des UMNS) fokussieren sollte und weniger auf die Spastik (Plussymptome des UMNS).

Bei allem sollte man die verschiedenen Facetten des motorischen Lernens berücksichtigen. Zum Beispiel die Verwendung realer Alltagsgegenstände (Bezug zur Transfertheorie), die Intensität (Repetitionen und Anstrengungsgrad), die kontinuierliche Anpassung des Trainings an das Leistungsniveau des Übenden (Shaping), seine Autonomie und die Verwendung eines externen Aufmerksamkeitsfokus (markierte Zielpunkte, klare Bewegungseffekte).

Repetitiv und intensiv üben $\rightarrow$ Damit das Flaschentraining gelingt, ist es ratsam, die Übungen zunächst unter Supervision zu lernen. Lernt der Patient die Übungen allein, so besteht die Gefahr, dass er einzelne Bewegungen kompensiert [4]. Anschließend führt er sie selbstständig als Eigenprogramm mehrmals wöchentlich durch, am besten täglich. Der Therapeut gestaltet die Übungen individuell für den Patienten, passt sie von Zeit zu Zeit an seinen aktuellen Leistungsstand an und kontrolliert sie in regelmäßigen Abständen. Der Patient sollte jede Übung mindestens 20 Mal wiederholen. Zur Motivation kann der Therapeut eine Tabelle mit Fotos, Zielsetzung und Wiederholungszahlen der Übungen sowie den Wochentagen erstellen, in die der Patient nach jeder Trainingseinheit einträgt. Um seine Autonomie zu unterstützen, kann er die Reihenfolge der Übungen in jedem Training selbst bestimmen.

Die abgebildeten Übungen stellen nur eine Auswahl dessen dar, was Patienten alles mit einer Flasche trainieren können. Wir erheben keinen Anspruch auf Vollständigkeit. Es werden Übungen für proximale und distale Schlüsselkomponenten und für unterschiedliche Leistungsniveaus gezeigt (schwere und moderate Armparese).

Martin Huber und Christine Horstmann

$\boldsymbol{\rightarrow}$ Literaturverzeichnis und Patienteninformation zum Download www.thieme-connect.de/products/physiopraxis > "Ausgabe 3/19“

\section{Hintergrundwissen}

\section{Schlüsselkomponenten}

Für das Training der oberen Extremität ist vor allem die Aktivierung folgender Bewegungskomponenten relevant [3]:

Reichen: (Transport der Hand zum Objekt)

$\rightarrow$ Schultergelenk: Flexion, Außenrotation

$\rightarrow$ Ellenbogen: Extension

$\rightarrow$ Handgelenk und Finger: Extension

$\rightarrow$ Daumen: Extension, Opposition

Greifen und Manipulieren: (Kontakt mit dem Objekt)

$\rightarrow$ Pronation und Supination

$\rightarrow$ Radialduktion und Ulnarabduktion

$\rightarrow$ Handgelenk: aktive (und passive) Extension

$\rightarrow$ lumbrikaler Griff (Flexion Grundgelenke)

$\rightarrow$ Finger: selektive Flexion und Extension

$\rightarrow$ Daumen: selektive Flexion und Adduktion sowie Extension und Abduktion 


\section{Dorsalextension}
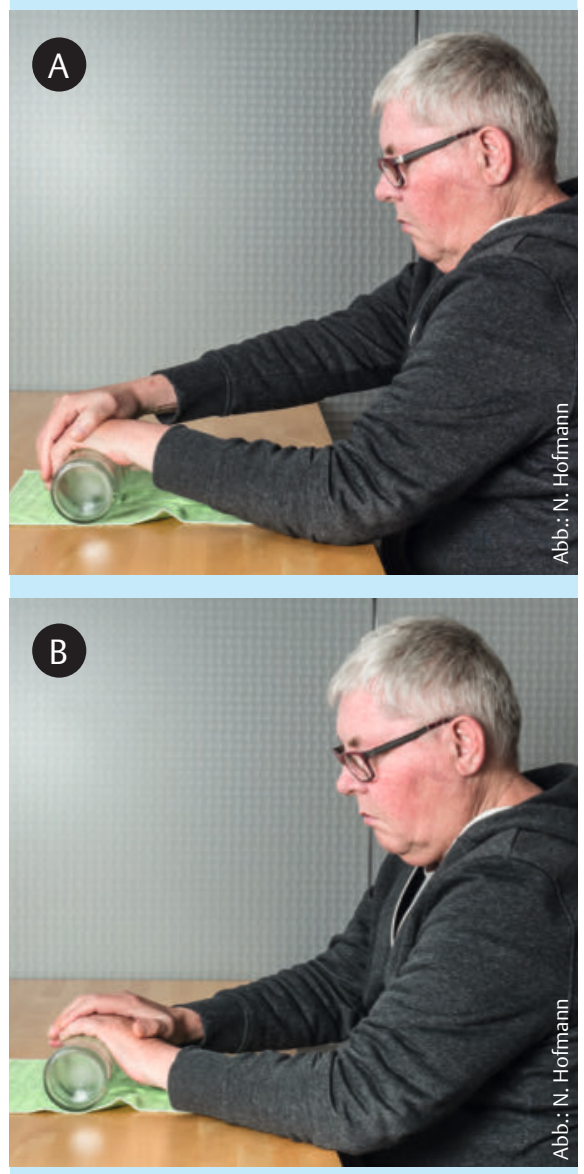

ASTE: Der Patient legt die Finger der linken Hand geöffnet auf den Flaschenbauch, der Daumen ist leicht abduziert. Das Handgelenk ist in Palmarflexion, das Ellenbogengelenk in Pronationsstellung. Die rechte Hand unterstützt die linke (॰ ABB. A).

Aktion: Der Patient rollt die Flasche zum Oberkörper hin, sodass im linken Handgelenk eine Dorsalextension entsteht (o ABB. B). Anschließend rollt der Patient die Flasche wieder zurück.

Ziel: Aktivierung der Schlüsselkomponente Dorsalextension des linken Handgelenks
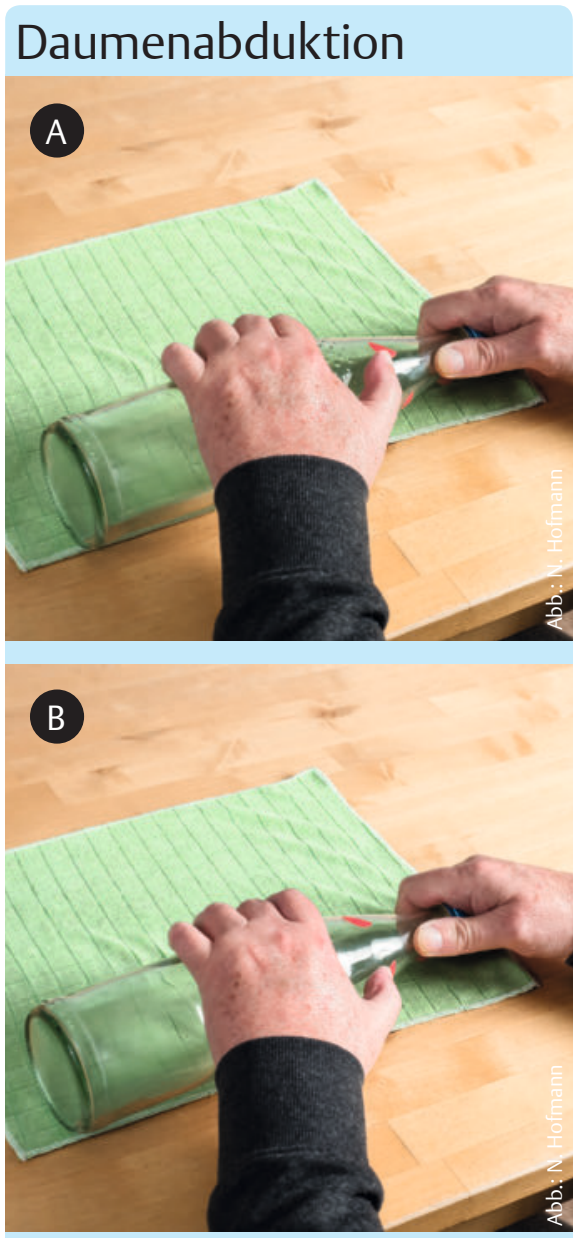

ASTE: Der Patient hält die liegende Flasche mit der linken Hand fest. Mit der rechten Hand stabilisiert er die Flasche. Zwei rote Punkte an der Flasche dienen als Zielpunkte für den Daumen. Diesen platziert er in relativer Adduktion auf dem oberen Punkt (○ ABB. A).

Aktion: Der Patient bewegt den linken Daumen vom oberen Punkt auf den unteren roten Punkt, indem er ihn abduziert (○ ABB. B). Anschließend bewegt er seinen Daumen wieder in die Ausgangsstellung zurück.

Ziel: Aktivierung der Schlüsselkomponente Daumenabduktion links
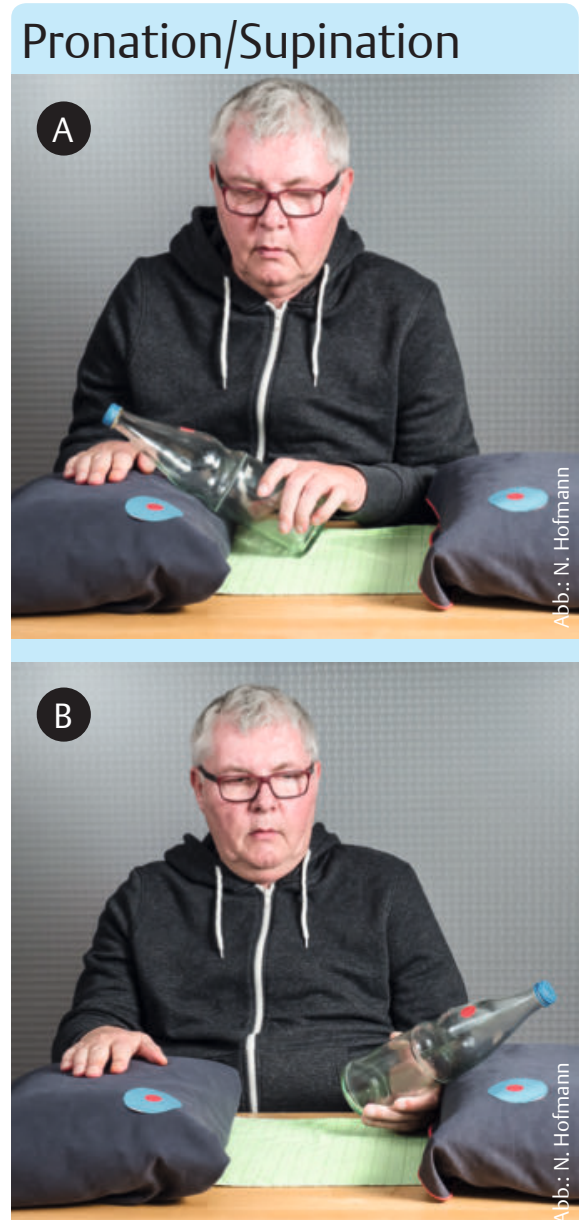

ASTE: Der Patient hält die stehende Flasche mit der linken Hand fest und kippt sie auf das rechte Kissen. Das Ellenbogengelenk ist in Pronation. Die rechte Hand liegt auf dem rechten Kissen. Die Kissen limitieren die Bewegung. Auf innen und an der Flasche ist je ein Zielpunkt angebracht (○ ABB. A).

Aktion: Der Patient bewegt die Flasche zum Zielpunkt auf das linke Kissen und legt sie ab. Das Ellenbogengelenk ist in Supination (○ ABB. B). Anschließend bewegt der Patient die Flasche wieder zurück auf das linke Kissen.

Ziel: Aktivierung der Schlüsselkomponente Pronation/Supination der linken Hand 

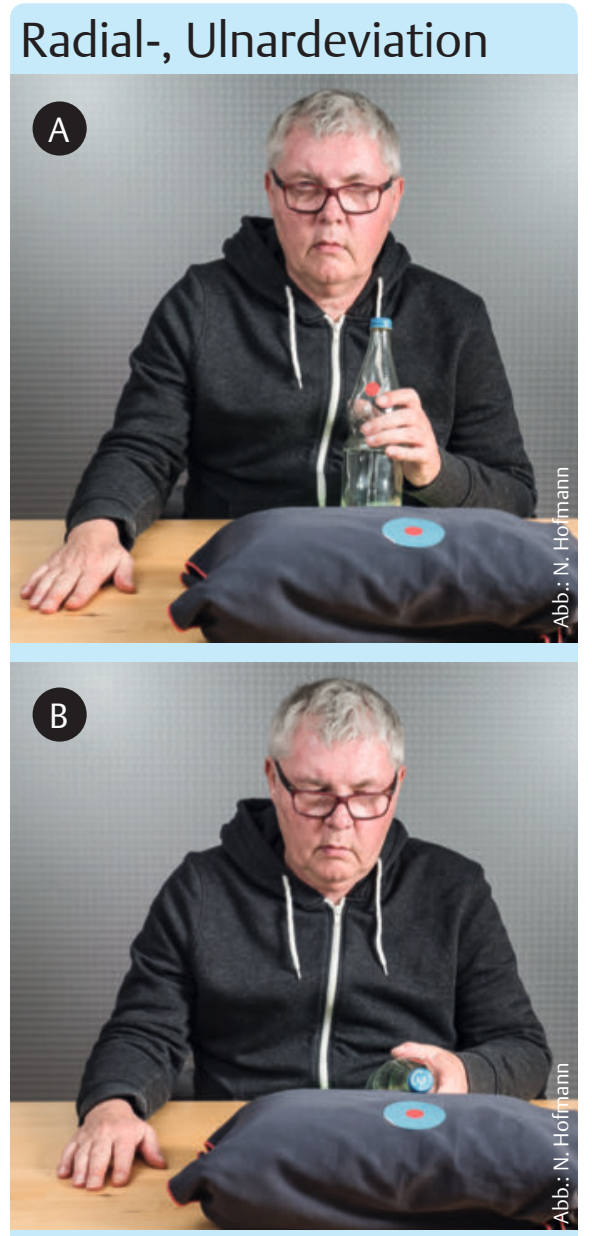

ASTE: Der Patient hält mit der linken Hand die stehende Flasche (○ ABB. A).

Aktion: Er kippt die Flasche zum Zielpunkt auf das Kissen (○ ABB. B). Die Zielpunkte sind einander angenähert. Das Handgelenk gelangt so in Dorsalextension und Ulnardeviation und das Ellenbogengelenk in relative Extension. Anschließend bewegt der Patient die Flasche zurück in die ASTE.

Ziel: Aktivierung der Schlüsselkomponente Radialduktion der linken Hand und Greifbewegung

\section{Flasche halten}
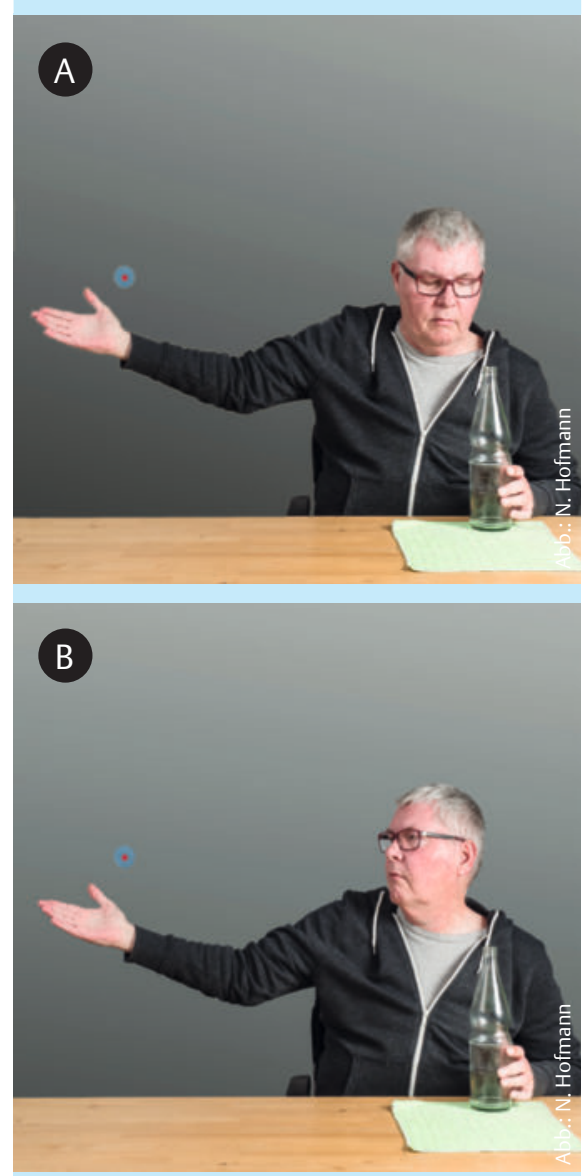

ASTE: Der Patient hält die aufgestellte Flasche mit der linken Hand, das Handgelenk ist in leichter Dorsalextension. Der Blick ist auf die Flasche gerichtet, die visuelle Kontrolle unterstützt das Halten der Flasche (○ ABB. A).

Aktion: Der Patient bewegt seinen rechten Arm nach rechts außen, die Augen sollen dieser Bewegung folgen und zum Zielpunkt an der Wand gucken. Die Flasche hält er dabei möglichst stabil (○ ABB. B). Anschließend geht sein Blick zurück zur Mitte, sein Arm bleibt auf der Seite. Nun bewegt er seinen Kopf erneut auf die Seite.

Ziel: Aktivierung der Schlüsselkomponente Dorsalextension im linken Handgelenk und Außenrotation im linken Schultergelenk unter Ausschluss der visuellen Kontrolle

\section{Außenrotation im SG}
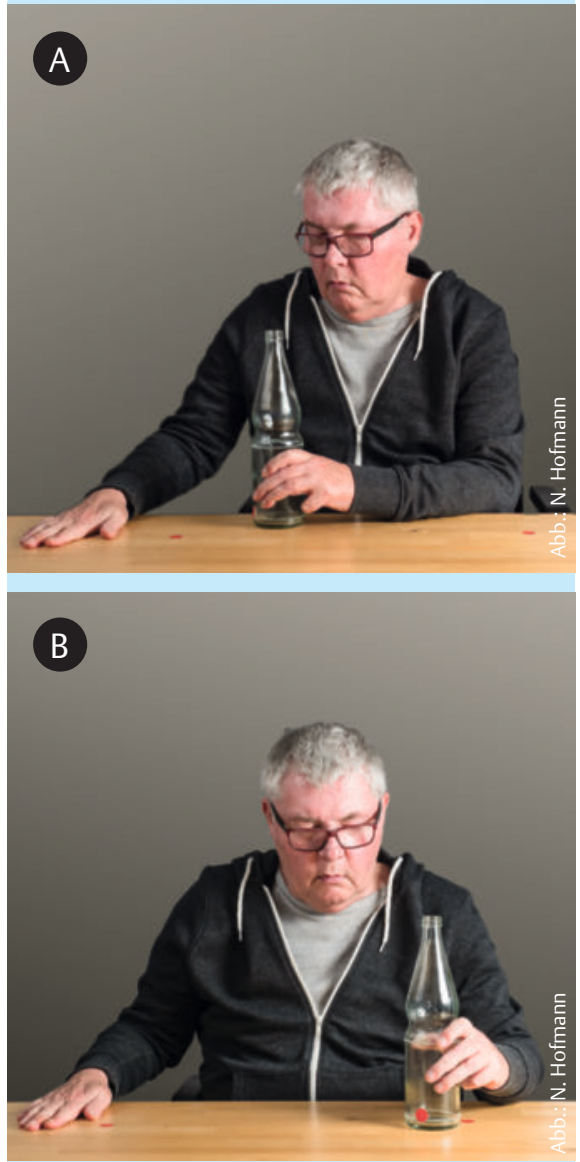

ASTE: Der Patient hält die aufgestellte Flasche mit der linken Hand am Flaschenbauch. Ein roter Punkt rechts auf der Tischplatte markiert, wo die Flasche zu Beginn stehen soll. Das Schultergelenk (SG) ist innenrotiert und adduziert. Das Ellenbogengelenk ist in Flexion und wenn möglich in einer Neutralstellung zwischen Pro- und Supination. Das Handgelenk ist in Dorsalextension. Der Patient umschließt die Flasche mit den Fingern (○ ABB. A).

Aktion: Der Patient transportiert die Flasche vom rechten Startpunkt zum Zielpunkt auf der linken Seite (o ABB. B). Das linke SG ist in 0-Stellung, das Ellenbogengelenk in Flexion und das Handgelenk in Dorsalextension. AnschlieBend bewegt der Patient seinen Arm wieder in die ASTE und wiederholt die Übung.

Ziel: Aktivierung der Schlüsselkomponente Außenrotation im linken Schultergelenk 

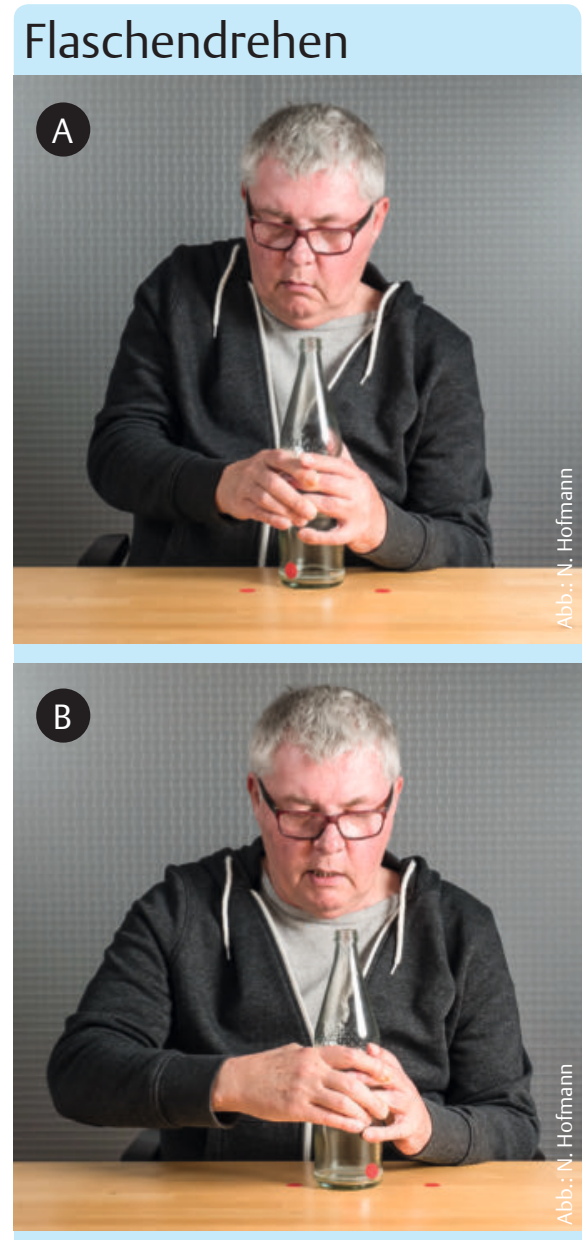

ASTE: Der Patient hält die aufgestellte Flasche mit der linken Hand, die rechte Hand unterstützt sie. Das linke Handgelenk ist in Palmarflexion. Der rote Punkt an der Flasche zeigt in Richtung des rechten Punkts auf dem Tisch (○ ABB. A).

Aktion: Der Patient rotiert die Flasche, sodass der Punkt auf der Flasche in Richtung des linken Punkts am Tisch gedreht wird (○ ABB. B). Das linke Handgelenk ist in Dorsalextension. Anschließend bewegt der Patient die Flasche in die ASTE und wiederholt die Übung.

Ziel: Aktivierung der Schlüsselkomponente Dorsalextension im linken Handgelenk
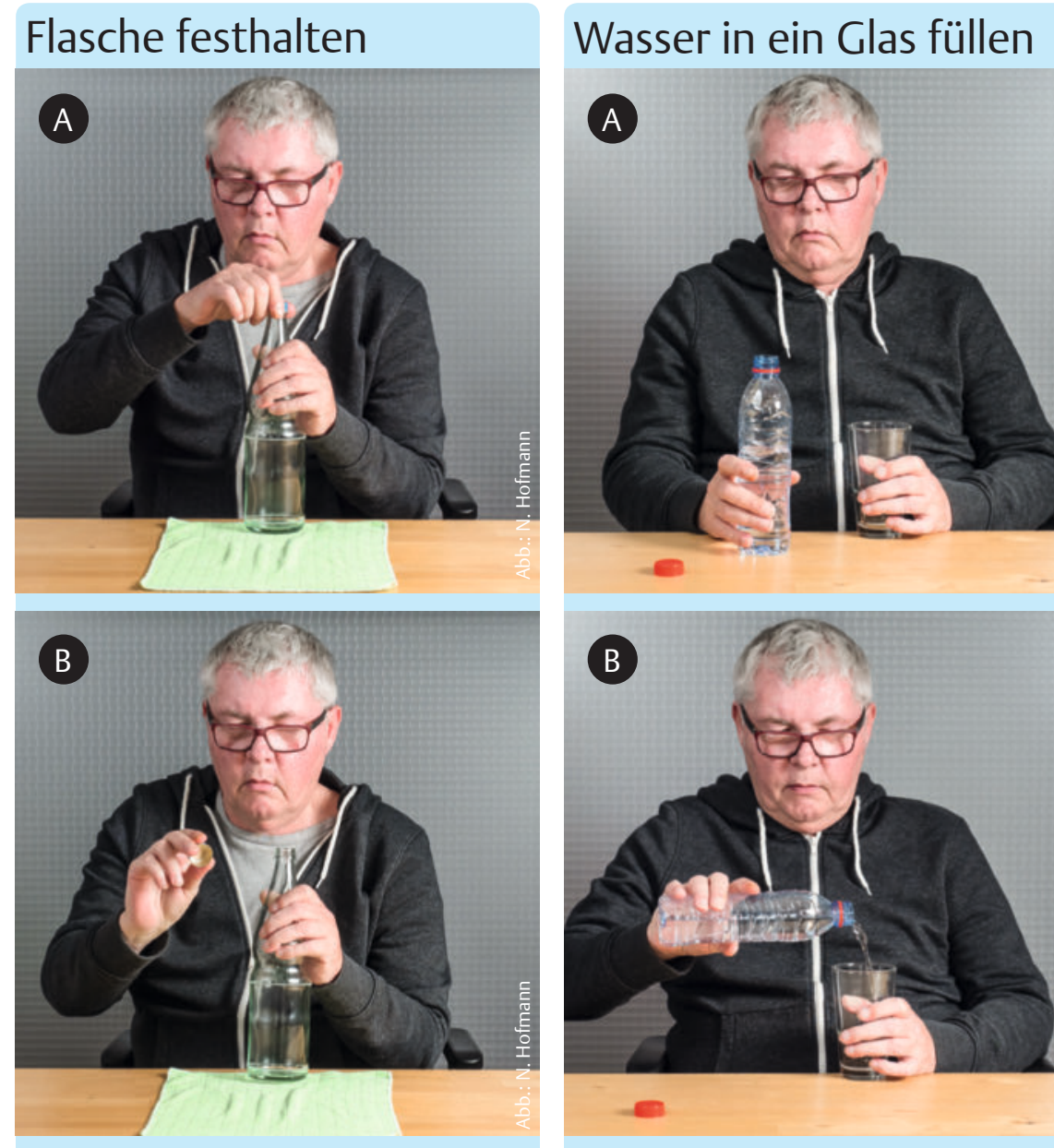

ASTE: Der Patient hält die aufgestellte Flasche mit der linken Hand am Flaschenhals oder Flaschenbauch (๑ ABB. A).

Aktion: Mit der rechten Hand öffnet er den anfangs nur leicht anschraubten Flaschenverschluss und legt ihn auf den Tisch (○ ABB. B). Ein feuchtes Tuch verhindert das Wegrutschen der Flasche. Anschließend schraubt der Patient den Verschluss wieder auf die Flasche und wiederholt die Übung.

Ziel: Aktivierung der Schlüsselkomponente lumbrikaler Griff der linken Hand und der Grobmotorik über die Haltefunktion
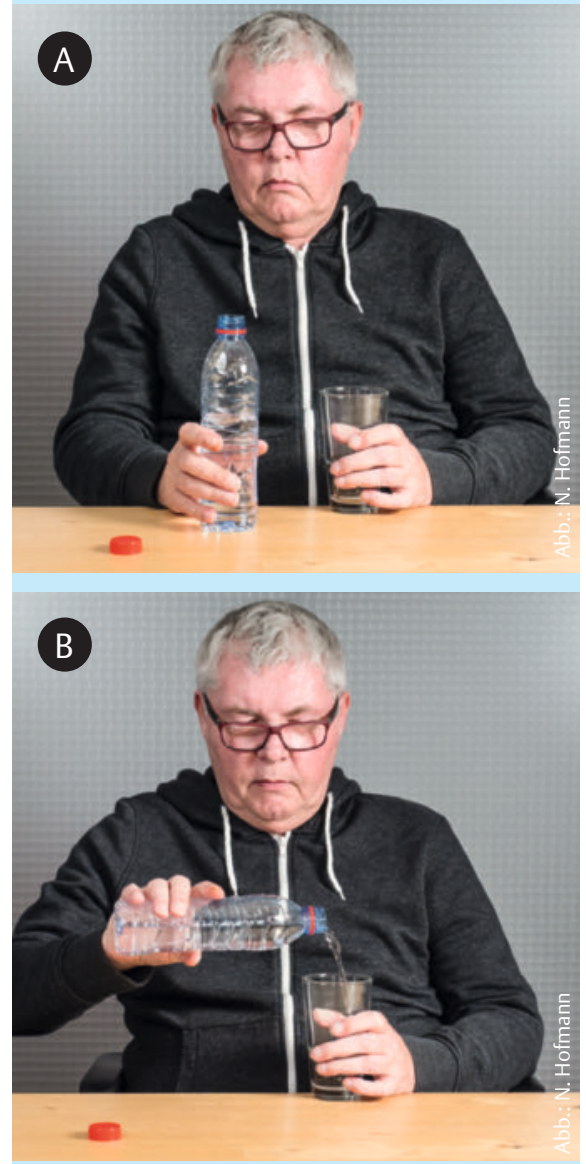

ASTE: Der Patient hält mit der linken Hand ein Glas und mit der rechten Hand die Flasche (॰ ABB. A).

Aktion: Langsam füllt er Wasser in das Glas (○ ABB. B).

Ziel: Aktivierung der Schlüsselkomponente Dorsalextension im linken Handgelenk, selektive Fingerflexion und der Greifbewegung der linken Hand 

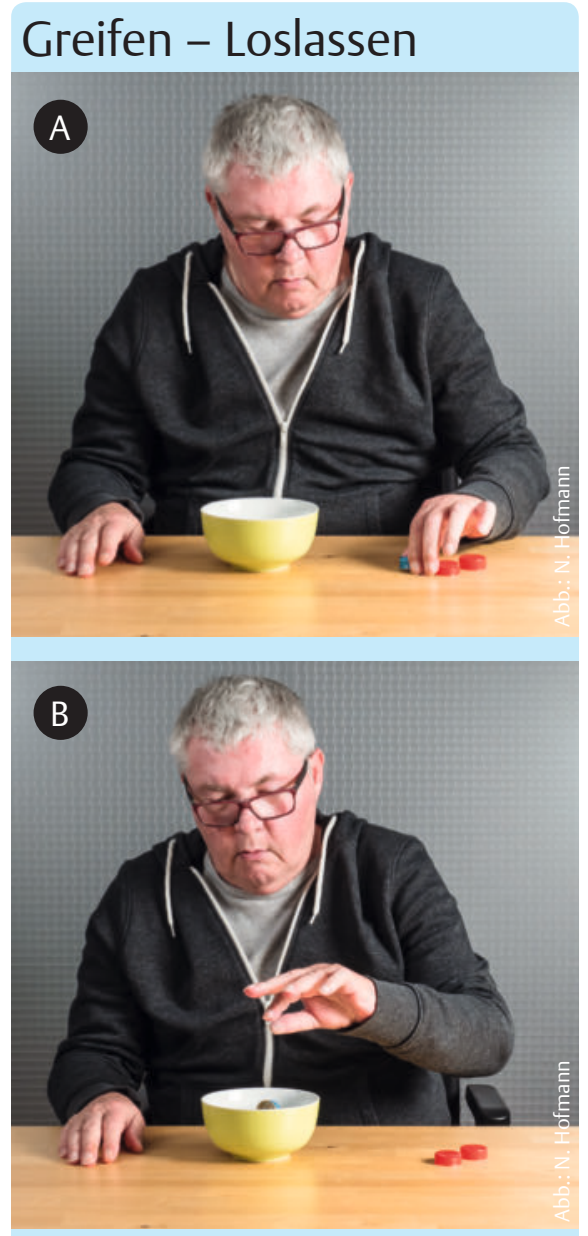

ASTE: Der Patient legt die rechte Hand auf den Tisch. Mit der linken Hand greift er nacheinander die Flaschendeckel, die auf dem Tisch liegen (○ ABB. A).

Aktion: Mit der lumbrikalen Grifformung für selektive Fingerbewegungen greift der Patient den Deckel, hebt den Unterarm an und bewegt die Hand über die Schale. Anschließend lässt er den Deckel los (॰ ABB. B).

Ziel: Aktivierung der Schlüsselkomponente lumbrikaler Griff und selektive Fingerbewegungen der linken Hand; SG-Flexion, Feinmotorik und Armtransport
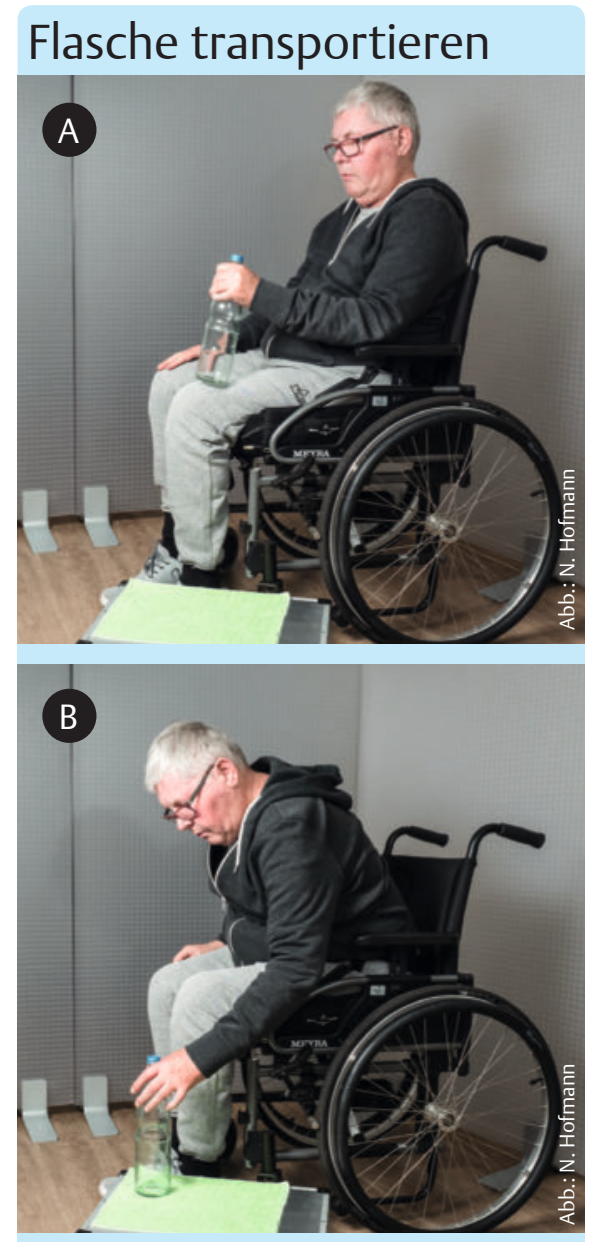

ASTE: Der Patient hält mit der linken Hand die Flasche, die vertikal auf dem linken Oberschenkel steht, die Finger sind in Flexion und das Handgelenk in Dorsalextension (○ ABB. A).

Aktion: Er hebt die Flasche an, transportiert sie zum Zielpunkt auf die Kiste und stellt sie dort ab (○ ABB. B). Der Oberkörper hat eine leichte Vorlage, sein SG ist links flektiert, sein Ellenbogengelenk und die Finger sind in Extension.

Ziel: Aktivierung der Schlüsselkomponente lumbrikaler Griff der linken Hand, SG-Flexion und Ellenbogenflexion; Feinmotorik und Armtransport
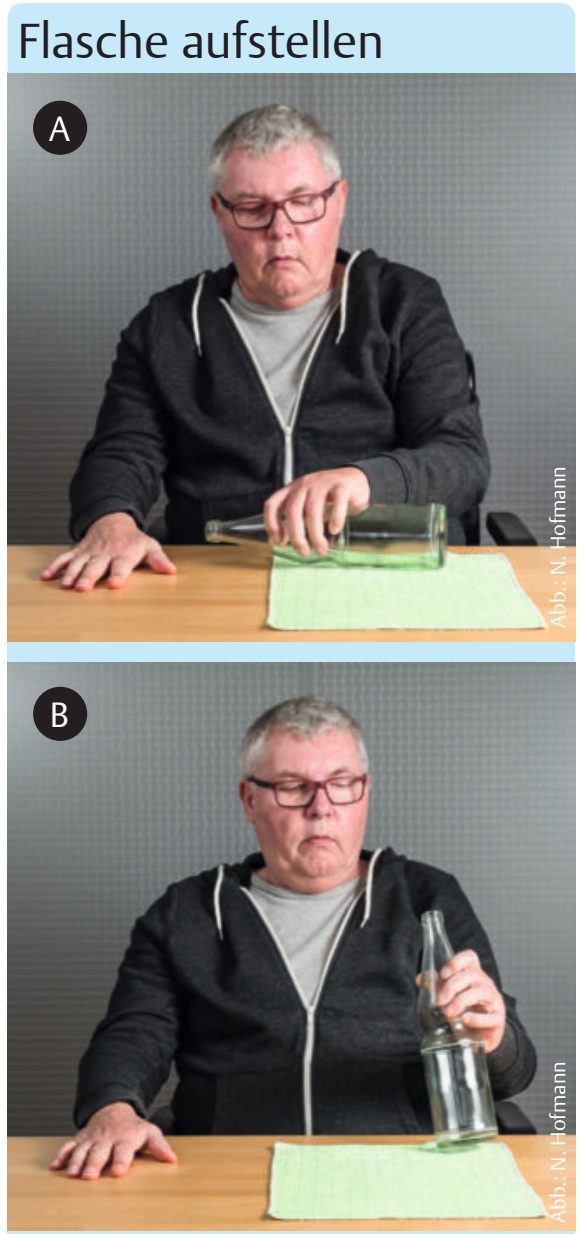

ASTE: Die Flasche liegt quer vor dem Patienten, die er mit der linken Hand hält. Das Ellenbogengelenk ist in Pronation. Das Handgelenk ist möglichst in Dorsalextension. Die rechte Hand liegt auf dem Tisch (o ABB. A).

Aktion: Der Patient stellt die Flasche auf (○ ABB. B). Der linke Ellenbogen ist in 0 -Stellung.

Ziel: Aktivierung der Schlüsselkomponenten Pronation/Supination der linken Hand 